\title{
LOCAL SKIN BUCKLING OF CYLINDRICAL SHELLS
}

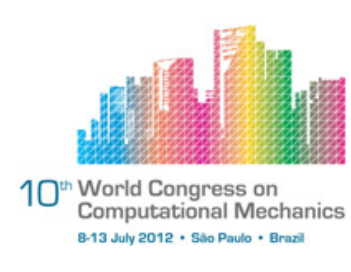

F. A. C. Monteiro, E. Lucena Neto, J. A. Hernandes

Instituto Tecnológico de Aeronáutica (corresponding-facm@ita.br)

\begin{abstract}
A formulation for local skin buckling of cylindrical shells is presented, where just a skin portion between adjacent stringers and frames is considered. The skin panel is subjected to both axial compressive and pressure loads. A buckling problem is formulated by a simplified linearization approach and its solution is obtained by a Galerkin procedure. A simple buckling formula is then derived and its accuracy is compared with finite element results.
\end{abstract}

Keywords: Local buckling, Cylindrical shells, Galerkin method.

\section{INTRODUCTION}

Stiffened panels are widely employed in aeronautical industry, when light weight aspects are essential, e.g. aircraft fuselages. Such structures are usually circular cylindrical and thin-walled susceptible to buckling.

A stiffened shell panel, depending on its geometry and stiffness, can exhibit different buckling modes which are neither mutually exclusive nor independent. For a panel subjected to uniaxial compression, like the ones found on the lower fuselage belly of commercial transport aircraft, the overall (global) buckling and the skin (local) buckling modes are usually distinct to each other. As sudden global buckling is undesirable at design limit load, typical designs exhibit skin buckling first, followed by load re-distribution to the stiffeners. Since the preliminary design phases where optimization iterations are performed, it is required the prediction of buckling load of the fuselage panels. For this purpose, the use of finite element method is usually avoided due to simulation time costs. Simple analytical buckling solutions provide, therefore, an important initial design tool.

In general, the buckling strength of a panel is evaluated assuming that the panel is simply supported or clamped on fuselage stiffeners (stringers and frames). However the local buckling strength should increase under the influence of the stiffener torsional rigidity. Since the stiffeners provide a continuous torsional rigidity along panel edges, they could be modeled as torsion bars. Such assumption seems to be correct if the stiffeners do not buckle before the skin buckling takes place, which is the case of a typical aeronautical design. Bearing this in mind, the stability behavior of the complete structure can be studied considering only the portion of a skin panel between adjacent stringers and frames, converting the complex original problem to the study of stability behavior of an individual skin panel elastically restrained along the edges. 
This key idea is exploited by several authors. For instance, Paik and Thayamballi [11] have employed the Lévy method to develop design formulations for buckling strength as a function of the torsional rigidity of support members that provide the rotational restraints along edges. In a simple manner, Bisagni and Vescovini [2] present an analytical formulation for the study of linearized local skin buckling load where the skin is modelled as a thin plate described by von Kármán theory and applying classical lamination approach, while the stringers are considered as torsion bars. Only axial compressive loads are involved.

A fast semi-analytical model for the postbuckling analysis of stiffened cylindrical panels is presented in [4]. The formulation includes both stringer and frames as structural elements, and captures their instabilities. It is an excelent study that leads to a consistent postbuckling behavior within an inherently complicated formulation. In a different pathway, following a simpler engineering approach, Pevzner et al. [13] develop some analytical formulae for calculating the collapse load of an axially compressed laminated curved panel, where torsional buckling and combined bending and torsion buckling of the stringers are included. However, in their approximate solution, the panel is assumed to be simply supported on stringers and clamped on frames. Therefore, the stringer torsional rigidity is not taken into account and the predicted buckling load may be somewhat inaccurate. A simple and accurate formulation for the buckling analysis of stiffened cylindrical panels have recently been proposed by [9] that extent the work of [2] to shell panels.

An extension of our previous formulation [9] is herein presented where the skin panel is now subjected to both axial compressive and pressure loads. It is assumed that the structure exhibits skin buckling first, which enables the local instability to be analyzed considering only a portion of a skin between two adjacent stringers and frames that give it support. Elastic rotational restraint is only provided by stringers. The equations to determine the bifucationpoint load is obtained through a simplified linearization of Donnell theory [3]. Based on appropriated trial mode functions of a single degree of freedom, the Galerkin type procedure is employed to seek a simple formula to estimate the local skin buckling load of stiffened cylindrical shells. The accuracy of the results is compared with those obtained using finite element models.

\section{PROBLEM FORMULATION}

Consider the circular cylindrical stiffened shell shown in Fig. 1, that represents a section of a traditional fuselage of commercial aircraft. It is assumed that:

- stringers are equally spaced;

- axial compressive loading $\bar{N}$ and lateral pressure $\bar{p}$ (difference between external and internal pressures) are uniformly applied;

- skin buckling occurs first.

Therefore, to access the fuselage buckling strenght it suffices to determine the critical buckling load of an individual panel, like the one highlighted in Fig. 1. The analyzed panel has length $a$, width $b$, thickness $h$, radius of curvature $R(R \gg h)$, and its middle surface is referred to a set of curvilinear coordinates $x y z$. It is considered that frames give simply support condition to the panel, while stringers provide some rotational restraint along edges. Figure 2 presents a schematic view of the assumed boundary conditions. 


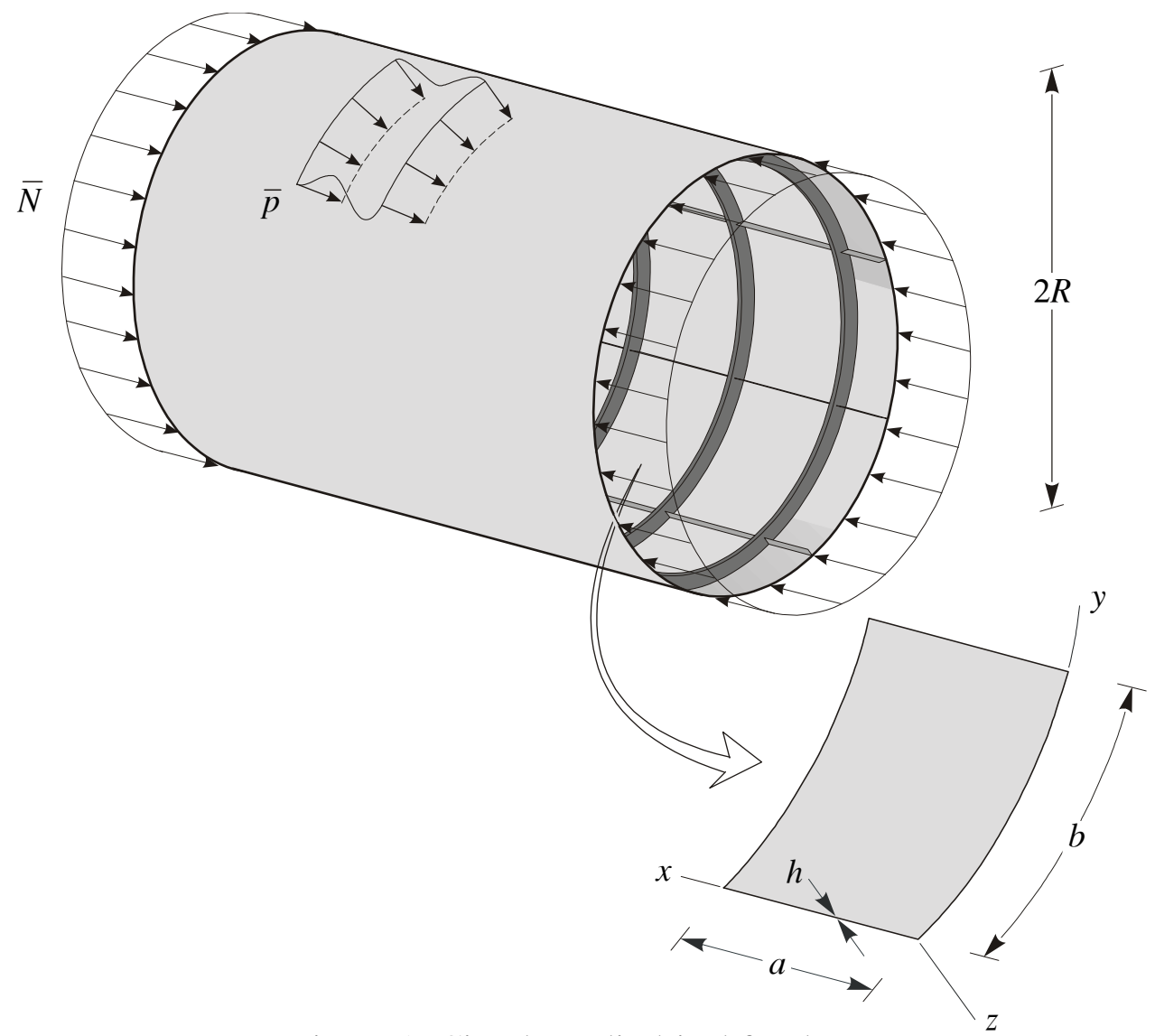

Figure 1. Circular cylindrical fuselage.

\subsection{Kinematic relations}

Consistent with the Kirchhoff-Love assumptions of a thin-shell theory, the following displacement field is assumed

$$
\begin{aligned}
& u_{x}(x, y, z)=u(x, y)-z w_{, x} \\
& u_{y}(x, y, z)=v(x, y)-z w_{, y} \\
& u_{z}(x, y, z)=w(x, y)
\end{aligned}
$$

in which $u, v, w$ are the displacements of a point $(x, y, 0)$ on the shell midsurface. The derivatives with respect to $x$ and $y$ coordinates are, respectively, denoted by ()$_{, x}$ and ()$_{, y}$.

Substituting the displacement field into the von Kármán-Donnell strain-displacement relations, we obtain the associated nonzero strains

$$
\epsilon_{x}=\epsilon_{x}^{m}+z \kappa_{x} \quad \epsilon_{y}=\epsilon_{y}^{m}+z \kappa_{y} \quad \gamma_{x y}=\gamma_{x y}^{m}+z \kappa_{x y}
$$

where

$$
\begin{aligned}
& \epsilon_{x}^{m}=u_{, x}+\frac{1}{2} w_{, x}^{2} \quad \epsilon_{y}^{m}=v_{, y}+\frac{w}{R}+\frac{1}{2} w_{, y}^{2} \quad \gamma_{x y}^{m}=v_{, x}+u_{, y}+w_{, x} w_{, y} \\
& \kappa_{x}=-w_{, x x} \quad \kappa_{y}=-w_{, y y} \quad \kappa_{x y}=-2 w_{, x y} .
\end{aligned}
$$

Note that the use of the circumferential coordinate $y$ instead of an angular coordinate facilitates comparisons between the shell and the equivalent plate expressions [5]. 


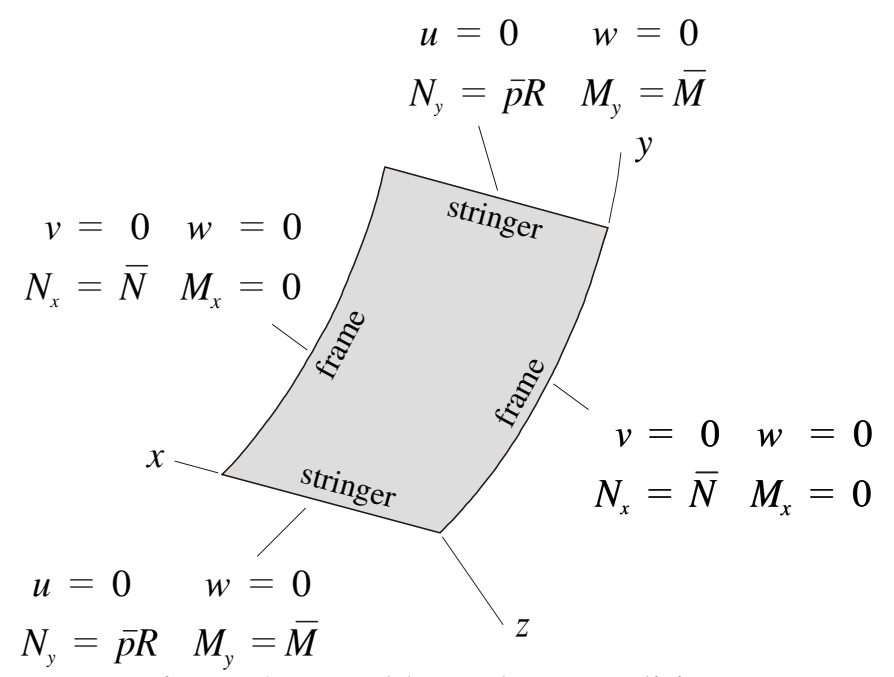

Figure 2. Panel boundary conditions.

\subsection{Equilibrium equations}

The use of the kinematic relations in the principle of virtual work yields the following Euler-Lagrange equations

$$
\begin{aligned}
N_{x, x}+N_{x y, y} & =0 \\
N_{x y, x}+N_{y, y} & =0 \\
M_{x, x x}+2 M_{x y, x y}+M_{y, y y}+N_{x} w_{, x x}+2 N_{x y} w_{, x y}+N_{y}\left(w_{, y y}-\frac{1}{R}\right)+\bar{p} & =0 .
\end{aligned}
$$

The panel boundary conditions are set in the form

$$
\begin{aligned}
& N_{x}=\bar{N} \quad v=0 \quad w=0 \quad M_{x}=0 \quad: x=0, a \\
& u=0 \quad N_{y}=\bar{p} R \quad w=0 \quad M_{y}=\bar{M} \quad: y=0, b
\end{aligned}
$$

with

$$
\bar{M}(x, 0)=\frac{G_{r} J_{r}}{K} w_{, x x y}(x, 0) \quad \bar{M}(x, b)=-\frac{G_{r} J_{r}}{K} w_{, x x y}(x, b)
$$

where $G_{r} J_{r}$ represents the stringer torsional rigidity and $K$ is a distribution factor. Since the stringers are equally spaced and all of them have the same geometry, it is straightforward to take $K=2$. The chosen boundary conditions Eq. (5) correspond to realistic support conditions, because the support given by the frames and stringers obviously prevent, respectively, circumferential and longitudinal displacements. However, the null moment condition $M_{x}=0$ is rather difficult to realize [7].

\subsection{Linearized buckling equations}

In the solution of equilibrium equations of a perfect shell panel subject to compressive stress state, it is observed the existence of an equilibrium path bifurcation in the vicinity of the undeformed configuration. To investigate the possible existence of adjacent-equilibrium configurations, it is common to give an arbitrarily small increments $u_{1}, v_{1}, w_{1}$ to the displacement variables and, then, examine equilibrium configuration represented by the displacements after the increment:

$$
u \rightarrow u_{0}+u_{1} \quad v \rightarrow v_{0}+v_{1} \quad w \rightarrow w_{0}+w_{1} .
$$


Fields $\left(u_{0}, v_{0}, w_{0}\right)$ and $(u, v, w)$ describe adjacent-equilibrium configurations associated with the primary (before increment) and secondary (after increment) equilibrium paths, respectively. In stability applications the displacement $\left(u_{0}, v_{0}, w_{0}\right)$ is called the prebuckling deformation and $\left(u_{1}, v_{1}, w_{1}\right)$ is called the buckling mode.

Accordingly, let

$$
\left\{\begin{array}{c}
N_{x} \\
N_{y} \\
N_{x y}
\end{array}\right\} \rightarrow\left\{\begin{array}{c}
N_{x}^{0} \\
N_{y}^{0} \\
N_{x y}^{0}
\end{array}\right\}+\left\{\begin{array}{c}
N_{x}^{1} \\
N_{y}^{1} \\
N_{x y}^{1}
\end{array}\right\} \quad\left\{\begin{array}{c}
M_{x} \\
M_{y} \\
M_{x y}
\end{array}\right\} \rightarrow\left\{\begin{array}{c}
M_{x}^{0} \\
M_{y}^{0} \\
M_{x y}^{0}
\end{array}\right\}+\left\{\begin{array}{c}
M_{x}^{1} \\
M_{y}^{1} \\
M_{x y}^{1}
\end{array}\right\}
$$

where the terms with 0 superscripts are associated to $u_{0}, v_{0}, w_{0}$ displacements, and the terms with 1 superscripts represent a portion of the incremental generalized stress that are linear in $u_{1}, v_{1}, w_{1}$.

Substitution of Eq. (8) into Eq. (4) yields two sets of equilibrium expressions. One of them is the nonlinear prebuckling problem:

$$
\begin{aligned}
& N_{x, x}^{0}+N_{x y, y}^{0}=0 \\
& N_{x y, x}^{0}+N_{y, y}^{0}=0 \\
& M_{x, x x}^{0}+2 M_{x y, x y}^{0}+M_{y, y y}^{0}+N_{x}^{0} w_{0, x x}+2 N_{x y}^{0} w_{0, x y}+N_{y}^{0}\left(w_{0, y y}-\frac{1}{R}\right)+\bar{p}=0 \\
& N_{x}^{0}=\bar{N} \quad v_{0}=0 \quad w_{0}=0 \quad M_{x}^{0}=0 \quad: x=0, a \\
& u_{0}=0 \quad N_{y}^{0}=\bar{p} R \quad w_{0}=0 \quad M_{y}^{0}=0 \quad: y=0, b .
\end{aligned}
$$

The other defines the so-called linearized buckling problem:

$$
\begin{aligned}
N_{x, x}^{1}+N_{x y, y}^{1} & =0 \\
N_{x y, x}^{1}+N_{y, y}^{1} & =0
\end{aligned}
$$

where $F_{0}$ and $F_{1}$ are given by

$$
\begin{aligned}
& F_{0}\left(w_{1}\right)=N_{x}^{0} w_{1, x x}+2 N_{x y}^{0} w_{1, x y}+N_{y}^{0} w_{1, y y} \\
& F_{1}\left(w_{0}\right)=N_{x}^{1} w_{0, x x}+2 N_{x y}^{1} w_{0, x y}+N_{y}^{1} w_{0, y y} .
\end{aligned}
$$

Note that lateral pression $\bar{p}$ is positive inward and, from boundary conditions Eq. (12) of the linearized problem, that stringers apply a torsional moment $\bar{M}$ to panel edges immediately after the onset of local buckling. 


\subsection{Constitutive relations}

Let skin material be defined by the elastic constants $E$ and $\nu$. The generalized stressstrain equation of the skin panel is defined by

$$
\begin{aligned}
& \left\{\begin{array}{c}
N_{x} \\
N_{y} \\
N_{x y}
\end{array}\right\}=\left[\begin{array}{ccc}
A_{11} & A_{12} & 0 \\
A_{12} & A_{22} & 0 \\
0 & 0 & A_{66}
\end{array}\right]\left\{\begin{array}{c}
\epsilon_{x} \\
\epsilon_{y} \\
\gamma_{x y}
\end{array}\right\}=\frac{E h}{1-\nu^{2}}\left[\begin{array}{ccc}
1 & \nu & 0 \\
\nu & 1 & 0 \\
0 & 0 & \frac{1-\nu}{2}
\end{array}\right]\left\{\begin{array}{c}
\epsilon_{x} \\
\epsilon_{y} \\
\gamma_{x y}
\end{array}\right\} \\
& \left\{\begin{array}{c}
M_{x} \\
M_{y} \\
M_{x y}
\end{array}\right\}=\left[\begin{array}{ccc}
D_{11} & D_{12} & 0 \\
D_{12} & D_{22} & 0 \\
0 & 0 & D_{66}
\end{array}\right]\left\{\begin{array}{c}
\kappa_{x} \\
\kappa_{y} \\
\kappa_{x y}
\end{array}\right\}=\frac{E h^{3}}{12\left(1-\nu^{2}\right)}\left[\begin{array}{ccc}
1 & \nu & 0 \\
\nu & 1 & 0 \\
0 & 0 & \frac{1-\nu}{2}
\end{array}\right]\left\{\begin{array}{c}
\kappa_{x} \\
\kappa_{y} \\
\kappa_{x y}
\end{array}\right\} .
\end{aligned}
$$

The constitutive relations presented herein are specialized to isotropic materials. However, they could be easily adapted for specially orthotropic laminates, e.g., regular symmetric cross-ply laminates.

\subsection{Simplified linearized buckling equations}

The extraction of the portion of the incremental generalized stress that are linear in $u_{1}, v_{1}, w_{1}$ could be obtained from the associated generalized stress increment as follows. Substituting Eq. (7) into Eq. (3) and considering the constitutive relations (14)

$$
\begin{aligned}
N_{x}= & A_{11}\left(u_{0, x}+\frac{1}{2} w_{0, x}^{2}\right)+A_{12}\left(v_{0, y}+\frac{w_{0}}{R}+\frac{1}{2} w_{0, y}^{2}\right) \\
& +A_{11}\left(u_{1, x}+w_{0, x} w_{1, x}+\frac{1}{2} w_{1, x}^{2}\right)+A_{12}\left(v_{1, y}+\frac{w_{1}}{R}+w_{0, y} w_{1, y}+\frac{1}{2} w_{1, y}^{2}\right)
\end{aligned}
$$

from which

$$
N_{x}^{1}=A_{11}\left(u_{1, x}+w_{0, x} w_{1, x}\right)+A_{12}\left(v_{1, y}+\frac{w_{1}}{R}+w_{0, y} w_{1, y}\right) .
$$

In a similar manner, it could be derived $N_{y}^{1}, M_{x}^{1}, M_{y}^{1}$ and $M_{x y}^{1}$, namely,

$$
\begin{aligned}
N_{y}^{1} & =A_{12}\left(u_{1, x}+w_{0, x} w_{1, x}\right)+A_{22}\left(v_{1, y}+\frac{w_{1}}{R}+w_{0, y} w_{1, y}\right) \\
N_{x y}^{1} & =A_{66}\left(v_{1, x}+u_{1, y}+w_{0, y} w_{1, x}+w_{0, x} w_{1, y}\right) \\
M_{x}^{1} & =-D_{11} w_{1, x x}-D_{12} w_{1, y y} \\
M_{y}^{1} & =-D_{12} w_{1, x x}-D_{22} w_{1, y y} \\
M_{x y}^{1} & =-2 D_{66} w_{1, x y} .
\end{aligned}
$$

Substituting Eqs. (16) and (17) into Eq. (11) and considering arbitrarily small increment displacements $\left(u_{1}, v_{1}, w_{1}\right) \ll 1$,

$$
\begin{gathered}
A_{11} u_{1, x x}+A_{12}\left(v_{1, x y}+\frac{w_{1, x}}{R}\right)+A_{66}\left(v_{1, x y}+u_{1, y y}\right)+\theta_{u}\left(w_{0}, w_{1}\right)=0 \\
A_{66}\left(v_{1, x x}+u_{1, x y}\right)+A_{12} u_{1, x y}+A_{22}\left(v_{1, y y}+\frac{w_{1, y}}{R}\right)+\theta_{v}\left(w_{0}, w_{1}\right)=0 \\
D_{0}\left(w_{1}\right)+\frac{A_{12}}{R} u_{1, x}+\frac{A_{22}}{R}\left(v_{1, y}+\frac{w_{1}}{R}\right)-F_{0}\left(w_{1}\right)+\theta_{w}\left(w_{0}, w_{1}\right)=0
\end{gathered}
$$


where $D_{0}, \theta_{u}, \theta_{v}$ and $\theta_{w}$ are given by

$$
\begin{aligned}
D_{0}\left(w_{1}\right) & =D_{11} w_{1, x x x x}+2\left(D_{12}+2 D_{66}\right) w_{1, x x y y}+D_{22} w_{1, y y y y} \\
\theta_{u}\left(w_{0}, w_{1}\right) & =A_{11}\left(w_{0, x} w_{1, x}\right)_{, x}+A_{12}\left(w_{0, y} w_{1, y}\right)_{, x}+A_{66}\left(w_{0, y} w_{1, x}+w_{0, x} w_{1, y}\right)_{, y} \\
\theta_{v}\left(w_{0}, w_{1}\right) & =A_{66}\left(w_{0, y} w_{1, x}+w_{0, x} w_{1, y}\right)_{, x}+A_{12}\left(w_{0, x} w_{1, x}\right)_{, y}+A_{22}\left(w_{0, y} w_{1, y}\right)_{, y} \\
\theta_{w}\left(w_{0}, w_{1}\right) & =\frac{1}{R}\left(A_{12} w_{0, x} w_{1, x}+A_{22} w_{0, y} w_{1, y}\right)-F_{1}\left(w_{0}\right) .
\end{aligned}
$$

The quantities $\theta_{u}, \theta_{v}, \theta_{w}$ are prebuckling rotation terms.

In order to solve the linearized buckling problem Eq. (18), in a practical manner, we make the following simplifications. Firstly, the prebuckling problem defined by Eqs. (9) and (10) is solved disregarding all flexural effects (the well-known linear membrane solution):

$$
N_{x}^{0}=\bar{N} \quad N_{x y}^{0}=0 \quad N_{y}^{0}=\bar{p} R .
$$

Thus,

$$
F_{0}\left(w_{1}\right)=\bar{N} w_{1, x x}+\bar{p} R w_{1, y y}
$$

or

$$
F_{0}\left(w_{1}\right)=\lambda\left(w_{1, x x}+\eta w_{1, y y}\right)
$$

where $\eta=\bar{p} R / \bar{N}$ is a nondimensional load parameter. When the panel is subject only to axial compression loading $\eta=0$, and when it is subject only to lateral pressure $\eta \rightarrow \infty$. The symbol $\lambda$ represents a load parameter which takes the value $\lambda \leftarrow \bar{N}$ when $\bar{N} \neq 0$ and $\lambda \leftarrow \bar{p} R$ when $\bar{N}=0$.

Secondly, the influence of rotation terms are thought negligible:

$$
\theta_{u} \rightarrow 0 \quad \theta_{v} \rightarrow 0 \quad \theta_{w} \rightarrow 0 .
$$

After having applied these assumptions the simplified linearized buckling equations, also known as Donnell stability equations in coupled form, is finally obtained

$$
\begin{gathered}
A_{11} u_{1, x x}+A_{12}\left(v_{1, x y}+\frac{w_{1, x}}{R}\right)+A_{66}\left(v_{1, x y}+u_{1, y y}\right)=0 \\
A_{66}\left(v_{1, x x}+u_{1, x y}\right)+A_{12} u_{1, x y}+A_{22}\left(v_{1, y y}+\frac{w_{1, y}}{R}\right)=0 \\
D_{0}\left(w_{1}\right)+\frac{A_{12}}{R} u_{1, x}+\frac{A_{22}}{R}\left(v_{1, y}+\frac{w_{1}}{R}\right)-F_{0}\left(w_{1}\right)=0
\end{gathered}
$$

or

$$
\left([E]-\lambda\left[E_{0}\right]\right)\{u\}=\{0\}
$$

where

$$
[E]=\left[\begin{array}{ccc}
E_{11}(\cdot) & E_{12}(\cdot) & E_{13}(\cdot) \\
E_{12}(\cdot) & E_{22}(\cdot) & E_{23}(\cdot) \\
E_{13}(\cdot) & E_{23}(\cdot) & E_{33}(\cdot)
\end{array}\right] \quad\left[E_{0}\right]=\left[\begin{array}{ccc}
0 & 0 & 0 \\
0 & 0 & 0 \\
0 & 0 & E_{00}(\cdot)
\end{array}\right] \quad\{u\}=\left\{\begin{array}{c}
u_{1} \\
v_{1} \\
w_{1}
\end{array}\right\}
$$

with

$$
\begin{array}{lll}
E_{11}(\cdot)=A_{11}(\cdot)_{, x x}+A_{66}(\cdot)_{, y y} & E_{12}(\cdot)=\left(A_{12}+A_{66}\right)(\cdot)_{, x y} & E_{13}(\cdot)=\frac{A_{12}}{R}(\cdot)_{, x} \\
E_{22}(\cdot)=A_{22}(\cdot)_{, y y}+A_{66}(\cdot)_{, x x} & E_{23}(\cdot)=\frac{A_{22}}{R}(\cdot)_{, y} & E_{33}(\cdot)=D_{0}(\cdot)+\frac{A_{22}}{R^{2}} \\
E_{00}(\cdot)=(\cdot)_{, x x}+\eta(\cdot)_{, y y} \cdot & &
\end{array}
$$

It is important to note that for a general investigation of influence of boundary conditions the set of Donnell equations in coupled form Eq. (24) should always be used [3]. 


\section{BUCKLING SOLUTION}

Longitudinal edges of the panel shown in Fig. 2 are elastically restrained, which can be considered as an intermediate condition between the simply supported and the clamped condition. Bearing this in mind, let the following trial mode function set:

$$
\{u\}=\left\{\begin{array}{c}
u_{1} \\
v_{1} \\
w_{1}
\end{array}\right\} \approx\left[\begin{array}{ccc}
X_{u} Y_{u} & 0 & 0 \\
0 & X_{v} Y_{v} & 0 \\
0 & 0 & X_{w} Y_{w}
\end{array}\right]\left\{\begin{array}{c}
U_{1} \\
V_{1} \\
W_{1}
\end{array}\right\}=[\Phi]\{D\}=\{U\}
$$

with

$$
\begin{aligned}
& X_{u}(x)=\cos \lambda_{a} x \quad X_{v}(x)=\sin \lambda_{a} x \quad X_{w}(x)=\sin \lambda_{a} x \\
& Y_{u}(y)=\sin \lambda_{b} y \quad Y_{v}(y)=\cos \lambda_{b} y \quad Y_{w}(y)=Y_{w, s}(y)+\vartheta Y_{w, c}(y)
\end{aligned}
$$

where $\lambda_{a}=m \pi / a$ and $\lambda_{b}=n \pi / b$. The quantities $U_{1}, V_{1}, W_{1}$ are unknown displacement amplitudes whereas $m, n$ represent the number of half waves in buckling mode in the longitudinal and in the circumferential direction, respectively. The transversal buckling shape along $y$-direction, namely, $Y_{w}$ is commonly described by the portions $Y_{w, s}$ and $Y_{w, c}$ that correspond to the limit cases of a simply supported stringer on the one hand (given by $Y_{w, s}$ ) and a rigidly clamped stringer on the other hand (given by $Y_{w, c}$ ). Of course, the actual situation of a stringer attached to skin will be found somewhere in between these two limit cases. The stringer torsional rigidity is introduced by $\vartheta$ parameter: when the stringer has no torsional rigidity $\vartheta=0$, and when it has infinite torsional rigidity $\vartheta \rightarrow \infty$.

It has been a common practice in literature to adopt $Y_{w}$ as superposition of a sine term with an undefined number of half waves and a cosine term with a single half wave $[2,6,9,12]$. However, this procedure lead to erroneous buckling solutions when pressure loads are involved due to use of a single half wave mode related to clamped conditions. To remedy this, the shape functions $Y_{w, s}$ and $Y_{w, c}$ are chosen as follows:

$$
Y_{w, s}(y)=\sin \lambda_{b} y \quad Y_{w, c}(y)=\sin \lambda_{b} y \sin \frac{\pi y}{b} .
$$

The former mode function describes the well-known pure rotational mode of the stringer cross-section. The latter mode function is picked from the hierachical set of trigonometric functions proposed in [1] that provides a physically meaningful buckling shape of a clamped stringer. From substitution of the trial function set Eq. (28) into the boundary conditions of the linearized buckling problem Eq. (12), considering Eq. (6) and Eq. (17) relations, a trial function set satisfying all boundary conditions are obtained if we establish

$$
\vartheta \leftarrow \frac{G_{r} J_{r}}{2 D_{22} K} \frac{b}{\pi} \lambda_{a}^{2}
$$

Now we substitute Eq. (28) into Eq. (25), and then we apply the Galerkin procedure to obtain

$$
\int_{0}^{a} \int_{0}^{b}\{U\}^{T}\left([E]-\lambda\left[E_{0}\right]\right)\{U\} d x d y=0
$$

or

$$
\{D\}^{T} \int_{0}^{a} \int_{0}^{b}[\Phi]\left([E]-\lambda\left[E_{0}\right]\right)[\Phi] d x d y\{D\}=0 .
$$


Since components of $\{D\}$ are arbitrary and independent,

$$
\left([G]-\lambda\left[G_{0}\right]\right)\{D\}=\{0\}
$$

where

$$
\begin{gathered}
{[G]=\int_{0}^{a} \int_{0}^{b}[\Phi][E][\Phi] d x d y=\left[\begin{array}{ccc}
G_{11} & G_{12} & G_{13} \\
G_{12} & G_{22} & G_{23} \\
G_{13} & G_{23} & G_{33}
\end{array}\right]} \\
{\left[G_{0}\right]=\int_{0}^{a} \int_{0}^{b}[\Phi]\left[E_{0}\right][\Phi] d x d y=\left[\begin{array}{ccc}
0 & 0 & 0 \\
0 & 0 & 0 \\
0 & 0 & G_{00}
\end{array}\right] .}
\end{gathered}
$$

The load parameter $\lambda$ corresponds to the eigenvalue of standard buckling eigenproblem defined by Eq. (34), whose third equation has been multiplied by -1 for symmetry purpose. Using a symbolic mathematical tool and after some algebra we achieve:

$$
\begin{aligned}
& G_{11}=-\frac{a b}{4}\left(A_{11} \lambda_{a}^{2}+A_{66} \lambda_{b}^{2}\right) \quad G_{12}=-\frac{a b}{4}\left(A_{12}+A_{66}\right) \lambda_{a} \lambda_{b} \quad G_{13}=A_{12} \frac{b}{\pi} \lambda_{a} \xi_{1} \\
& G_{22}=-\frac{a b}{4}\left(A_{66} \lambda_{a}^{2}+A_{22} \lambda_{b}^{2}\right) \quad G_{23}=A_{22} \frac{b}{\pi} \lambda_{b} \xi_{1} \\
& G_{33}=-\left(\frac{A_{22}}{R^{2}}+D_{11} \lambda_{a}^{4}\right) \frac{b}{\pi} \xi_{2}-2\left(D_{12}+2 D_{66}\right) \frac{\pi}{b} \lambda_{a}^{2} \xi_{3}-D_{22} \frac{\pi^{3}}{b^{3}} \xi_{4} \\
& G_{00}=\frac{b}{\pi} \lambda_{a}^{2} \xi_{2}+\eta \frac{\pi}{b} \xi_{3}
\end{aligned}
$$

where

$$
\begin{aligned}
& \xi_{1}=\frac{a}{4 R}\left(\frac{8 n^{2}}{4 n^{2}-1} \vartheta+\pi\right) \\
& \xi_{2}=\frac{a}{8}\left(\pi \vartheta^{2}+\frac{32 n^{2}}{4 n^{2}-1} \vartheta+2 \pi\right) \\
& \xi_{3}=\frac{a}{8}\left[\left(n^{2}+1\right) \pi \vartheta^{2}+\frac{32 n^{4}}{4 n^{2}-1} \vartheta+2 n^{2} \pi\right] \\
& \xi_{4}=\frac{a}{8}\left[\left(n^{4}+6 n^{2}+1\right) \pi \vartheta^{2}+16\left(\frac{2 n^{4}}{4 n^{2}-1}+1\right) n^{2} \vartheta+2 n^{4} \pi\right] .
\end{aligned}
$$

From the assumption that a nontrivial solution of Eq. (34) exist, the critical buckling load $\lambda_{c r}$ is finally derived in the form

$$
\lambda_{c r}=\min _{m, n} \lambda(m, n)=\min _{m, n} \frac{\|[G]\|}{\left(G_{12}^{2}-G_{11} G_{22}\right) G_{00}} \quad m, n=1,2, \ldots
$$

For particular values of $a, b, h$ and $R$, the pair $(m, n)$ corresponding to the smallest eigenvalue $\lambda$ may be determined by trial. The resulting minimum eigenvalue is an upper bound to the actual critical-load parameter. The derivation of the linear critical load shown above is not of a general validity. Within the framework of a classical theory, the proposed buckling formula were derived assuming (i) shallowness of the shell surface; (ii) null torsion rigidity of frames. Inclusion of such features will be a meaningful improvement for current formulation. 


\section{RESULTS AND DISCUSSIONS}

The buckling load expression Eq. (38) is implemented in a MATLAB language computer program obtaining a fast design tool. A series of one-bay stiffened cylindrical shells with different number of equally spaced stringers are modeled and analyzed using solution SOL 105 (eigenvalue buckling analysis) of the finite element commercial code NASTRAN [10]. In the finite element modelling one considers that boundary edges of the stiffened cylindrical shell are simply supported by the frames. Also, no initial imperfections are introduced into the models.

Computations are carried out for 12 stiffened cylindrical shells as reported in Table 1, considering different geometries to evaluate the effect of different number of stiffeners $n_{r}$ and different aspect ratios $a / b$, with $b=2 \pi R / n_{r}$.

Table 1. Panel geometry

\begin{tabular}{ccccc}
\hline Panel ID & $a(\mathrm{~mm})$ & $R(\mathrm{~mm})$ & $h(\mathrm{~mm})$ & $\mathrm{n}^{\circ}$ stringers \\
\hline 1 & 400 & 2000 & 1 & 24 \\
2 & 400 & 2000 & 1 & 12 \\
3 & 400 & 1000 & 1 & 24 \\
4 & 400 & 1000 & 1 & 12 \\
5 & 600 & 2000 & 1 & 24 \\
6 & 600 & 2000 & 1 & 12 \\
7 & 600 & 1000 & 1 & 24 \\
8 & 600 & 1000 & 1 & 12 \\
9 & 800 & 2000 & 1 & 24 \\
10 & 800 & 2000 & 1 & 12 \\
11 & 800 & 1000 & 1 & 24 \\
12 & 800 & 1000 & 1 & 12 \\
\hline
\end{tabular}

Table 2. Stiffener cross-sectional dimensions

\begin{tabular}{cccc}
\hline \multicolumn{2}{c}{ Width $(\mathrm{mm})$} & \multicolumn{2}{c}{ Thickness $(\mathrm{mm})$} \\
\hline$w_{a}$ & 19.05 & $t_{a}$ & 1.27 \\
$w_{b}$ & 19.05 & $t_{b}$ & 1.27 \\
$w_{c}$ & 5.50 & $t_{c}$ & 3.00 \\
\hline
\end{tabular}

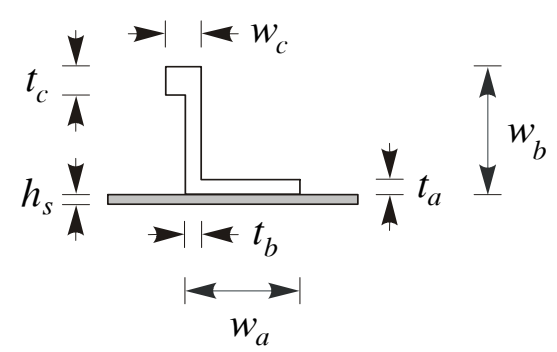

Figure 3. Stiffener section.

It is considered only stiffeners with $\mathrm{Z}$ cross-section type connected to the skin by friction stir welding process. Table 2 presents the cross-sectional dimensions of the Z-stiffener 
shown in Fig. 3. The stringer has a torsional constant $J_{r}=57.412 \mathrm{~mm}^{4}$. Panel skin and stringers are made of aluminum alloys commonly used in aeronautical applications, with mechanical properties described in Table 3. Finite element modeling follows the recommendations presented in [7]. Except the stringer upper flange, which is modeled using the simple beam element CBAR, the rest of panel structure are meshed with quadrilateral plate elements CQUAD4. The stiffened panel is discretized into sufficient number of elements to catch buckling modes correctly. A quadrialteral $4 \times 40$ element mesh is employed along the stringer web connected flange and at the underneath skin, whereas a $16 \times 40$ element mesh is employed between stringers. No attempt was made to optimise the mesh.

Table 3. Material properties

\begin{tabular}{llcc}
\hline Material & \multicolumn{1}{c}{ Type } & $E\left(\mathrm{~N} / \mathrm{mm}^{2}\right)$ & $\nu$ \\
\hline Skin & AL 2024-T3 & 72400 & 0.33 \\
Stiffener & AL 7050-T3511 & 71020 & 0.33 \\
\hline
\end{tabular}

So far we have assumed that stiffeners remain straight until the skin buckles. This assumption is normally appropriate for practical purpose, but they may in some cases distort before the buckling onset so that the stringers will not fully contribute to the rotational restraints along the edges. The effective torsional rigidity $G_{r} J_{r} / K$ should then be corrected by means of the related reduction factor

$$
\frac{G_{r} J_{r}}{K} \leftarrow C \frac{G_{r} J_{r}}{K}
$$

where $C$ is the reduction constant accounting for the distortion of stringers. For instance, Paik and Thayamballi [11] assume that $C$ is constant during the loading process and is proportional to the relative torsional rigidity between stringer and panel skin, as follows

$$
C=\frac{3 J_{r}}{b h^{3}} \quad \text { if } \quad J_{r} \leq \frac{b h^{3}}{3} \quad \text { or } \quad C=1.0 \quad \text { if } \quad J_{r}>\frac{b h^{3}}{3}
$$

whose validity will to some extent be confirmed by comparison with finite element solutions.

\subsection{Axial Compression}

As a first example let us consider a stiffened cylindrical shell that is simply supported at its ends and subjected to uniformly distributed compressive load $\bar{N}=1.0 / 2 \pi R[\mathrm{~N} / \mathrm{mm}]$, applied over skin panel. This case implies $\bar{p}=0$ and $\lambda \leftarrow \bar{N}$. The load parameter $\eta$ is null and the component $G_{00}$ reads $(b / \pi) \lambda_{a}^{2} \xi_{2}$. The critical values predicted by the proposed formula Eq. (38) are compared with those obtained from the finite element analysis (FEA) in Table 4. It can be observed that average difference from finite element results are $5 \%$, approximately.

\subsection{Uniform Lateral Pressure}

As a second example we consider a stiffened cylindrical shell that is simply supported at its ends and subjected to uniform lateral pressure $\bar{p}=1.0\left[\mathrm{~N} / \mathrm{mm}^{2}\right]$, positive inward. In this case $\bar{N}=0$ and $\lambda \leftarrow \bar{p} R$. The load parameter $\eta$ takes an undefined value and the component $G_{00}$ symplifies to $(\pi / b) \xi_{3}$. The critical values predicted by the proposed formula Eq. (38) are compared with those obtained from the FEA in Table 5. It can be observed that average difference from finite element results are $3 \%$. 
Table 4. Comparison between analytical formulation and FEA: axial compression

\begin{tabular}{|c|c|c|c|c|c|}
\hline \multirow[t]{2}{*}{ Panel ID } & \multicolumn{2}{|c|}{$(m, n)$} & \multicolumn{2}{|c|}{$\bar{N}_{c r}$} & \multirow[t]{2}{*}{$\mid$ Diff. $\mid \%$} \\
\hline & Eq. (38) & FEA & Eq. (38) & FEA & \\
\hline 1 & $(5,1)$ & $(5,3)$ & 21.96 & 23.18 & 5.29 \\
\hline 2 & $(5,1)$ & $(5,1)$ & 21.85 & 22.39 & 2.43 \\
\hline 3 & $(7,1)$ & $(7,1)$ & 44.34 & 49.21 & 9.91 \\
\hline 4 & $(7,1)$ & $(7,1)$ & 43.61 & 45.68 & 4.53 \\
\hline 5 & $(7,1)$ & $(1,2)$ & 22.11 & 23.51 & 5.93 \\
\hline 6 & $(8,1)$ & $(8,1)$ & 21.93 & 22.51 & 2.56 \\
\hline 7 & $(10,1)$ & $(2,2)$ & 44.25 & 48.51 & 8.79 \\
\hline 8 & $(11,1)$ & $(1,3)$ & 43.66 & 44.05 & 0.88 \\
\hline 9 & $(10,1)$ & $(1,2)$ & 21.96 & 21.37 & 2.75 \\
\hline 10 & $(10,1)$ & $(10,1)$ & 21.85 & 22.42 & 2.56 \\
\hline $11^{\dagger}$ & $(13,1)$ & $(3,2)$ & 44.33 & 49.69 & 10.78 \\
\hline $12^{\dagger}$ & $(14,1)$ & $(14,1)$ & 43.61 & 47.18 & 7.58 \\
\hline
\end{tabular}

${ }^{\dagger}$ Panels for which the first buckling mode is of global type: tabled results are concerned to the first local mode.

\section{CONCLUSIONS}

An approximated analytical solution for local skin buckling load of stiffened cylindrical shells is obtained based on Galerkin method. The strucure is subjected to both axial compressive and pressure loads. Analytical solutions are compared with those obtained using finite element models. For the analyzed shells it was found that:

- differences between FEA and proposed formula are, in an average sense, lower than $5 \%$ or $3 \%$ if the shell is subjected to axial compression or to uniform lateral pressure, respectively;

- number of half waves obtained analytically and numerically are almost identical.

The proposed solution gives a first insight on local skin buckling and could be easily implemented in a computer program. It seems to provide an effective preliminary design tool, when the analysis of different configurations is necessary.

\section{Acknowledgements}

The authors wish to express their gratitude to undergraduate student Paulo de Tarso Machado Leite Soares - Asp Of for his helpfull support in obtaining finite element solutions. 
Table 5. Comparison between analytical formulation and FEA: uniform lateral pressure

\begin{tabular}{|c|c|c|c|c|c|}
\hline \multirow[t]{2}{*}{ Panel ID } & \multicolumn{2}{|c|}{$(m, n)$} & \multicolumn{2}{|c|}{$\bar{p}_{c r} R$} & \multirow[t]{2}{*}{ |Diff.| \% } \\
\hline & Eq. (38) & FEA & Eq. (38) & FEA & \\
\hline 1 & $\overline{(1,3)}$ & $\overline{(1,3)}$ & 4.77 & 4.87 & 2.09 \\
\hline 2 & $(1,7)$ & $(1,6)$ & 4.64 & 5.08 & 8.52 \\
\hline 3 & $(1,2)$ & $(1,2)$ & 6.55 & 6.54 & 0.10 \\
\hline 4 & $(1,4)$ & $(1,4)$ & 6.40 & 6.44 & 0.74 \\
\hline 5 & $(1,3)$ & $(1,3)$ & 3.00 & 3.11 & 3.66 \\
\hline 6 & $(1,5)$ & $(1,5)$ & 2.93 & 3.12 & 6.26 \\
\hline 7 & $(1,2)$ & $(1,2)$ & 4.78 & 4.85 & 1.49 \\
\hline 8 & $(1,3)$ & $(1,3)$ & 4.11 & 4.15 & 1.03 \\
\hline 9 & $(1,2)$ & $(1,2)$ & 2.41 & 2.46 & 2.09 \\
\hline 10 & $(1,5)$ & $(1,5)$ & 2.09 & 2.26 & 7.13 \\
\hline $11^{\dagger}$ & $(1,2)$ & $(1,2)$ & 4.33 & 4.40 & 1.63 \\
\hline 12 & $(1,3)$ & $(1,3)$ & 2.94 & 3.00 & 2.22 \\
\hline
\end{tabular}

${ }^{\dagger}$ Panels for which the first buckling mode is of global type: tabled results are concerned to the first local mode.

\section{REFERENCES}

[1] Beslin O., Nicolas J., "A hierarchical functions set for predicting very high order plate bending modes with any boundary conditions". J. Sound Vibr. 202, 633-655, 1997.

[2] Bisagni C., Vescovini R., "Analytical formulation for local buckling and post-buckling analysis of stiffened laminated panels". Thin-Walled Struct. 47, 318-334, 2009.

[3] Brush D. O., Almorth B. O., Buckling of bars, plates, and shells. McGraw-Hill, 1975.

[4] Buermann P., Rolfes R., Tessmer J., Schagerl M., "A semi-analytical model for local postbuckling analysis of stringer- and frame-stiffened cylindrical panels". Thin-Walled Struct. 44, 102-114, 2006.

[5] Chajes A., Principles of structural stability theory. Prentice-Hall, 1974.

[6] Fujikubo M., Yao T., "Elastic local buckling strength of stiffened plate considering plate/stiffener interaction and welding residual stress". Marine Struct. 12, 543-564, 1999.

[7] Kollár L., Dulácska E., Buckling of shells for engineers. John Wiley, 1984.

[8] Lucena Neto E., Monteiro F. A. C., Ruela H. H., "A comparison of buckling performance of rivet and friction stir welding stiffened panels". In: Proceedings of the XXXI Iberian Latin-American Congress on Computational Methods in Engineering, 2010.

[9] Monteiro F. A. C., Lucena Neto E., Hernandes J. A., "Local skin buckling of fuselages". In: Proceedings of the XXXII Iberian Latin-American Congress on Computational Methods in Engineering, 2011.

[10] MSC, Quick Reference Guide. MSC Software Corporation, 2008.

[11] Paik J. K., Thayamballi A. K., "Buckling strength of steel plating with elastically restrained edges”. Thin-Walled Struct. 37, 27-55, 2000.

[12] Peres G., Lucena Neto E., Monteiro F. A. C., "Flambagem local de placas reforçadas". In: Proceedings of the XXXII Iberian Latin-American Congress on Computational Methods in Engineering, 2011 [Portuguese].

[13] Pevzner P., Abramovich H., Weller T. "Calculation of the collapse load of an axially compressed laminated composite stringer-stiffened curved panel - An engineering approach". Compos. Struct. 83, 341-353, 2008. 